\title{
Le cause per la dimissione dallo stato clericale secondo le competenze della Congregazione per la Dottrina della Fede
}

\section{DISMISSAL FROM THE CLERICAL STATE ACCORDING TO THE COMPETENCE OF THE CONGREGATION FOR THE DOCTRINE OF THE FAITH}

The author of the presented study carried out an analysis of the canonical regulations concerning the penalty of dismissal from the clerical state, according to the norms established by the document De Gravioribus Delictis. The article enumerates the more grave canonical delicts, which are associated with the penalty of dismissal and reserved to the exclusive competence of the Congregation for the Doctrine of the Faith. The first paragraph describes delicts against the Faith and the unity of the Church such as apostasy, heresy and schism. The second one refers to offences involving usurpation of ecclesiastical offices and abuse of the sacred power of ordination in exercise of it, called also as offences against the sanctity of the Holy Sacraments. The last paragraph presents more grave delicts against morality associated with the penalty of dismissal from the clerical state.

Key words: dismissal from the clerical state, more grave delicts, profanation, solicitation, abuse of the sacred power of ordination

Il can.1362 $\$ 1$ CIC e anche l'art. 42 della Costituzione Apostolica Pastor Bonus menzionano i delitti riservati alla competenza della CDF 
ma non ne danno un elenco ${ }^{1}$. Questo è stato fatto il 30 aprile 2001 con la promulgazione delle Norme de gravioribus delictis in forza del Motu Proprio di Giovanni Paolo II Sacramentorum Sanctitatis tutela. Tali norme sono state modificate il 21 maggio 2010 da Benedetto XVI.

Lo scopo di questo articolo non sarà semplicemente più profonda l'analisi di tutti i delitti più gravi riservati alla CDF. In questo articolo saranno contemplati solo quelli, che prevedono la possibilità di pena la dimissione dallo stato clericale quando il loro autore è un chierico. Le Norme de gravioribus delictis riservano per questa congregazione anche nuove azioni contro la fede, i sacramenti e i costumi. Gli altri delitti sottoposti a questa pena nella legge sono giudicati secondo le norme processuali codiciali.

\section{I delitti contro la religione e l'unità della Chiesa}

\section{Lapostasia, l'eresia e lo scisma}

Il primo gruppo di delitti contenuti nelle Norme de gravioribus delictis dal 2010 che prevedono la dimissione dallo stato clericale (cfr. can. $1364 \$ 2$ CIC) sono i delitti contro la fede (delicta contra fidem), tra i quali si distingue l'apostasia, l'eresia e lo scisma².

Il primo delitto contro la fede è l'apostasia. Essa si manifesta con un atto formale che attesta il rifiuto intenzionale della fede e l'abbandono della Chiesa. Consiste in «un ripudio totale della fede cristiana, sia in foro esterno che interno, fatto con un atto positivo della volontà da una persona battezzata» (cfr. can. $751 \mathrm{CIC}$ ). Esso può essere provocato ad esempio dall'adesione ad un'altra religione o ad ideologie come l'ateismo, l'agnosticismo o altre dottrine non compatibili con la fede cattolica. L'atto d'apostasia configura sempre un'eresia totale, in cui elemento costitutivo è un'ostinazione. Di conseguenza un apostata non può essere riconosciuto cristiano, a causa della negazione totale delle verità della fede ${ }^{3}$. «Come un atto di apostasia non si riconosce l'indifferenza religiosa o la mancata pratica della fede» ${ }^{4}$.

1 Cfr. Giovanni Paolo II, Costituzione Apostolica Pastor Bonus, 28 giugno 1988, AAS 80 (1988), art. 42.

2 Cfr. Congregazione per la Dottrina della Fede, Normae de gravioribus delictis Congregationi pro Doctrina Fidei reservatis seu Normae de delictis contra fidem necnon de gravioribus delictis, 21 maggio 2010, AAS 102 (2010), art. $1 \S 1$; art. 2 \$1.

$3 \quad$ Cfr. J. Syryjczyk, Apostazja od wiary w świetle przepisów kanonicznego prawa karnego. Studium prawno-historyczne, Warszawa 1984, p. 92.

$4 \quad$ M. Ferrante, Il delitto del apostasia alla luce del Motu Proprio "Omnium in mentem", [in:] AA.VV., Questioni attuali di diritto penale canonico, Studi Giuridici XCVI, Città del Vaticano 2012, p. 232. 
Il delitto successivo contro la fede è l'eresia, ed è definita come «ostinata negazione, dopo aver ricevuto il battesimo, di una qualche verità che si deve credere per fede divina e cattolica, o il dubbio ostinato su di essa» (Can. 751). Leretico non rifiuta tutta la Rivelazione Divina che la Chiesa impone di credere, sia dal Magistero solenne della Chiesa, sia dal suo magistero ordinario e universale ${ }^{5}$ (cfr. can. 750 §1). «L'eresia non è conosciuta come obiezione alle conclusioni teologiche, le quali non sono definite della Chiesa come verità rivelate. Inoltre non costituiscono eresia le negazioni o i dubbi di una verità che appartiene al deposito della fede, quando viene dal fatto da ignoranza o incomprensione ${ }^{6}$. L'elemento costitutivo è un'ostinazione, sulla base della quale si può constatare che l'atto eretico manifestato è collegato con la consapevole e volontaria azione che rifiuta una determinata verità di fede. La dichiarazione di tale ostinazione testimonia il fatto di commettere eresia non soltanto in forma occulta, ma anche pubblica.

Lultimo delitto contra fidem è lo scisma, conosciuto come «il rifiuto della sottomissione al Sommo Pontefice o della comunione con i membri della Chiesa a lui soggetti» (Can. 751 CIC). Lo scisma, a differenza dell'eresia e dell'apostasia, non è costituito direttamente dalla negazione della verità di fede. Il suo elemento costitutivo è la negazione del primato del Romano Pontefice o di altre cause riguardanti direttamente l'unità della Chiesa cattolica ${ }^{7}$. Spesso la conseguenza esterna di tale procedimento è l'abbandono della comunità ecclesiale nella quale il Romano Pontefice è riconosciuto come Capo e fondamento di unità. Tipico atto scismatico è la fondazione di una comunità religiosa che non riconosce il primato del Vescovo di Roma, collegato con la consacrazione episcopale $^{8}$ (cfr. can. 1382 CIC).Non costituisce scisma la sola disubbidienza al Romano Pontefice che non nega il suo primato o non

$5 \quad$ Cfr. D. Cito, Delicta graviora contro la fede e sacramenti, [in:] AA.VV., Questioni attuali di diritto penale canonico, Studi Giuridici XCVI, Città del Vaticano 2012, p. 35 .

$6 \quad$ B. F. Pighin, Diritto penale canonico, Venezia 2014, p. 306.

7 Cfr. J. Syryjczyk, Kanoniczne prawo karne. Część szczegótowa, Warszawa 2003, p. 21.

8 Il 30 luglio 1988 Mons. M. Lefebvre ha commesso un atto di natura scismatica, consacrando quali vescovi senza mandato pontificio quattro sacerdoti, incorrendo per questo nella scomunica latae sententiae in forza dei cann. 1382 e 1364 CIC; cfr. Congregazione dei Vescovi, Dichiarazione della scomunica di mons. M. Lefebvre, EV 11 (1988-1989), 692-694; cfr. V. De Paolis, D. Cito, Le sanzioni nella Chiesa. Commento al Codice di Diritto Canonico. Libro VI, Città del Vaticano 2000, p. 296. 
riguarda le cause direttamente legate all'unità della Chiesa9. Anche l'appartenenza alle associazioni che agiscano contro la Chiesa, come ad esempio la massoneria, non è ritenuta delitto conto la fede. Tuttavia considerando contraddizione fra le regole della massoneria ed il Magistero della Chiesa e la fede che la Chiesa professa, l'appartenenza alle cosiddette organizzazioni in alcuni casi può portare in effetto ad eresia, apostasia o scisma ${ }^{10}$.

I delitti di apostasia, eresia o scisma risultano commessi quando l'autore esprime consapevolmente un atto contro la fede o contro l'unita della Chiesa. Questo può succedere per scritto, in forma orale, tramite gesti o altri comportamenti. Però non deve considerarsi effettivamente compiuto, se nessuno raccoglie quella dichiarazione o manifestazione (cfr. can. 1330 CIC). Inoltre una condizione necessaria per l'esistenza di un delitto contra fidem è l'ostinazione dell'autore. La dichiarazione dell'ostinazione è necessaria nel caso di eresia e anche per questo l'apostasia è sempre eresia. Invece nel caso dello scisma il can. 751CIC non si esprime sul tema della ostinazione. Secondo alcuni canonisti l'ostinazione è un attributo dello scisma ${ }^{11}$. Lo scismatico ostinato può essere punito con le altre pene a norma del can. $1364 \$ 1$, non escludendo la dimissione dallo stato clericale (can. $1364 \S 2$ ).

\section{Lasportazione o la conservazione a scopo sacrilego delle specie consacrate, o la loro profanazione}

Il primo reato contro la santità dell'Eucaristia riservato alla CDF è «il delitto dell'asportazione o della conservazione a scopo sacrilego delle specie consacrate, o la loro profanazione» ${ }^{12}$. L'autore di tali delitti incorre nella scomunica latae sententiae iservata alla Sede Apostolica. Inoltre, quando è chierico può essere punito anche con un'altra pena ferendae sententiae, non escludendo dimissione dallo stato clericale (cfr. can. 1367 CIC).

9 Cfr. A. Marzoa, Commento al c. 1364, [in:] A. Marzoa, J. Miras, R. Rodríguez-Ocaña (a cura di), Comentario exegético al Código de Derecho Canónico, vol. IV/1, Pamplona 2002, p. 476.

10 Cfr. Congregazione per la Dottrina della Fede, Declaratio de associationibus massonicis, 26 novembre 1983, AAS 76 (1984) 300; cfr. C. Dezzuto, Delicta reservata contro la fede e contro $i$ sacramenti, [in:] AA.VV., I delitti riservati alla Congregazione per la Dottrina della Fede, Quaderni di Ius Missionale, Città del Vaticano 2014, p. 75-76.

11 Cfr. J. Syryjczyk, Kanoniczne prawo karne, 30.

12 Congregazione per la Dottrina della Fede, Normae de gravioribus delictis, art. 3 $\S 1,1^{\circ}$. 
Oggetto dei tre atti di sacrilego dell'Eucaristia sono soltanto le specie consacrate validamente ${ }^{13}$, indipendentemente dal rito in cui è stata fatta la consacrazione e anche dal fatto che il consacratore sia apostata, eretico o scismatico ${ }^{14}$, o sacerdote che, impedito per legge canonica, non può lecitamente celebrare l'Eucaristia (cfr. can. 900 §2 CIC). Loggetto di questo delitto sono le due specie eucaristiche oppure una di loro, in tutto o solo in una piccola parte di essa.

Un elemento essenziale del delitto dell'asportazione delle specie consacrate è la loro consapevole detenzione o asportarli a scopo sacrilego da un luogo dove sono conservate secondo le norme della legge. Questo avrà luogo sia quando esse saranno trasferite ad un'altra persona che sta per fare un atto sacrilego, sia quando sono conservate ad finem sacrilegum, indipendentemente dal fatto che in futuro, questo fine sarà realizzato o un meno ${ }^{15}$.

La seconda forma di insulto dell'Eucaristia viene conosciuta come conservazione fuori luogo previsto dalla legge canonica, ad esempio in una borsa, in tasca, a casa o in un altro luogo a scopo sacrilego ${ }^{16}$. Elemento essenziale del delitto è l'intenzione dell'autore; non si può parlare invece di delitto, nel caso di conservazione illecita, ciò non è soggetto a sanzioni penali (cfr. can. 1367 CIC).

Il terzo tipo di insulto grave all'Eucaristia, secondo l'art. $3 \S 1,1^{\circ}$ delle Norme de gravioribus delictis, la sua profanazione. ${ }^{17}$ Essa si configura come la dispersione delle ostie consacrate, o come spargimento del Sangue del Signore con odio, ira o disprezzo di $\mathrm{Dio}^{18}$.

Linsulto delle specie eucaristiche mediante asportazione, conservazione a scopo sacrilego o la profanazione è un delitto grave riservato alla Santa Sede (cfr. can. 1367 CIC). L'eventuale assoluzione dalla pena

$13 \quad$ Una persona che profana le specie non ancora consacrate pensando che sono già consacrate, commette soltanto un peccato grave secondo la intenzione della profanazione. Non commette il reato, quando profana le specie eucaristiche realmente consacrate avendo convinzione che loro non sono ancora consacrate; cfr. J. Syryjczyk, Profanacja Eucharystii wedtug ustawodastwa kanonicznego i polskiego prawa karnego, «Prawo Kanoniczne» 29 (1986), n. 3-4, p. 194.

14 Cfr. V. De Paolis, D. Cito, Le sanzioni nella Chiesa, p. 305.

15 Cfr. M. Stokłosa, Ochrona Najświętszej Eucharystii w świetle motu proprio Jana Pawta II Sacramentorum santitatis tutela, «Studia Redemptorystowskie» 8 (2010), p. 207.

16 Cfr. T.Pawluk, Prawo kanoniczne wedtug Kodeksu Jana Pawła II, vol. IV, Olsztyn 1990, p. 128.

17 Cfr. L. Chiappetta, Il Codice di Diritto Canonico. Commento giuridico-pastorale, vol. I-III, Bologna 2011, p. 701.

18 Cfr. J. Syryjczyk, Kanoniczne prawo karne, p. 48. 
della scomunica latae sententiae in foro interno spetta alla Penitenziaria Apostolica, invece in foro esterno alla CDF.

\section{Usurpazione degli uffici ecclesiastici e delitti nel loro esercizio}

\section{Lattentata azione liturgica del Sacrificio eucaristico}

Al giudizio della $\mathrm{CDF}$ è riservato anche il delitto dell'attentata azione liturgica del Sacrificio eucaristico di cui al can. 1378 § 2 n. 1 del CIC ${ }^{19}$.

Il can. $900 \S 1 \mathrm{CIC}$ dice, che «ministro, in grado di celebrare nella persona di Cristo il sacramento dell'Eucaristia, è il solo sacerdote validamente ordinato». Perciò «sono 'incapaci' di mettere in atto la sinassi eucaristica sia fedele ordinato diacono, che non è configurato ontologicamente con il sacerdozio ministeriale istituito da Cristo e che non ha l'abilità di agire in persona Christi capitis, sia gli altri fedeli, laici o di vita consacrata che siano, pur essendo investiti del servizio comune in forza del battesimo» ${ }^{20}$.

La persona che attenta azione liturgica del Sacrificio eucaristico non essendo sacerdote commette il reato ${ }^{21}$. Questo avviene quando l'autore vestito con i paramenti liturgici, va verso l'altare in modo che sembri apprestarsi a celebrare, recitando e compiendo i gesti almeno del canone eucaristico ${ }^{22}$. Allora commette il reato non soltanto chi celebra tutta la Santa Messa, ma anche soltanto la parte principale, a partire dal Prefazio fino alla preghiera Padre Nostro ${ }^{23}$. Il CIC nel can. 1378 $\S 2$ n. 1 stabilisce, che chi non elevato all'ordine sacerdotale attenta l'azione liturgica del Sacrificio eucaristico, incorre nella pena latae sententiae dell'interdetto, o, se chierico, della sospensione, cui può essere anche punito con la pena della dimissione dallo stato clericale.

19 Cfr. Congregazione per la Dottrina della Fede, Normae de gravioribus delictis, art. $3 \S 1,2^{\circ}$.

20 B. F. Pighin, Diritto penale canonico, p. 402.

21 Cfr. M. Wronowska, Przyczyny i procedura wydalenia ze stanu duchownego, «Studia Ełckie» 16 (2014), n. 4, p. 503.

22 Cfr. B. F. Pighin, Diritto penale canonico, p. 403.

23 Cfr. M. Wronowska, Przyczyny i procedura, p. 504. 


\section{Lattentata assoluzione sacramentale o l'ascolto vietato della confessione}

Secondo il can. $965 \mathrm{CIC}$ «Ministro del sacramento della penitenza è il solo sacerdote». Per la validità del sacramento, cioè validità dell'assoluzione dei peccati si richiede che il ministro, oltre alla potestà di ordine, abbia la facoltà di esercitarla sui fedeli ai quali imparte l'assoluzione (cfr. can. $966 \S 1 \mathrm{CIC}$ ).

La norma del can. 1378 \$2 n. 2 CIC prevede due reati, i quali possono essere commessi conto la santità del sacramento della penitenza: attentare all'amministrazione del sacramento della penitenza, o ascoltare la confessione sacramentale non avendo la capacità richiesta per farlo validamente. «Si tratta di attentata assoluzione sacramentale o ascolto vietato della confessione da parte di persona non abile, ossia da parte di chi non ha ricevuto l'ordine sacro e perciò non è in grado di impartire una valida assoluzione o un valido ascolto delle confessioni» ${ }^{24}$.

Questo avviene quando il soggetto inizia ad ascoltare la confessione sacramentale ossia la confessione del penitente diretta all'assoluzione sacramentale, e anche quando, non potendo amministrare validamente l'assoluzione sacramentale, pure cerca di impartirla.

Secondo la norma del can. $1378 \S 2$ CIC, il sacerdote che commette questo reato incorre nella scomunica latae sententiae riservata alla Sede Apostolica., non escludendo la dimissione dallo stato clericale. Il tribunale competente per giudicare la causa è $\mathrm{CDF}$, secondo art. 4 $\S 1$, n. 2 delle Normae.

\section{Lattentata ordinazione sacra di una donna}

Come è noto, il conferimento dell'ordine mediante imposizione delle mani e recita della preghiera prevista dai libri liturgici (cfr. can. 1009 $\$ 2 \mathrm{CIC}$ ) è riservato ai vescovi consacrati (cfr. can. $1012 \mathrm{CIC})$. La norma del can. 1024 CIC stabilisce che riceve validamente la sacra ordinazione esclusivamente il battezzato di sesso maschile. La Chiesa cattolica si riferisce negativamente la pratica dell'ordinazione delle donne, ciò che viene ammesso dalle altre Chiese cristiane. La posizione della Chiesa si basa sulla tradizione e sulla natura dell'ordinazione. La tradizione esclude chiaramente la possibilità di ordinazione delle donne, e la natura del sacramento del sacerdozio richiede, come soggetto, una persona di sesso maschile perché i sacramenti sono celebrati in persona Christi. Inoltre, secondo il Concilio Vaticano II è necessario distinguere chiaramente il sacerdozio ministeriale o gerarchico tra

$24 \quad$ D. Cito, Delicta graviora, p. 45. 
sacerdozio comune dei fedeli. «Il sacerdote ministeriale compie il sacrificio eucaristico con la potestà sacra di cui è investito, come se fosse Cristo stesso e lo offre a Dio a nome di tutto il popolo. Invece i fedeli esercitano il loro sacerdozio col ricevere i sacramenti, con la preghiera e il ringraziamento, con la testimonianza di una vita santa, con l'abnegazione e la carità operosa» ${ }^{25}$.

L'ultima opinione della Chiesa è stata ufficialmente espressa nella Lettera Apostolica di Giovanni Paolo II Ordinatio Sacerdotalis dal 22 maggio 1994, dove il Romano Pontefice ha dichiarato con fermezza che «la Chiesa non ha in alcun modo la facoltà di conferire alle donne l'ordinazione sacerdotale e che questa sentenza deve essere tenuta in modo definitivo da tutti i fedeli della Chiesa» ${ }^{26}$.

Le Norme de gravioribus delictis nell'art. 5 n. 3 alla CDF riservano anche il delitto più grave di attentata sacra ordinazione di una donna. «Secondo il disposto del can. 1378 CIC sia colui che attenta il conferimento del sacro ordine, sia la donna che ne attenta la ricezione, incorrono nella scomunica latae sententiae riservata alla Sede Apostolica ${ }^{27}$. Se poi il reo è un chierico, può essere punito con la dimissione o la deposizione.

Si deve sostenere che la donna è assolutamente incapace di ricevere l'ordine sacerdotale. Nel caso di eventuale conferimento dell'ordine sacro ad una donna, non si tratta di invalidità ma di inesistenza di queste ordinazioni.

\section{La consacrazione a fine sacrilego di una specie eucaristica o di entrambe nella Messa o fuori di essa}

Alla CDF è riservato anche il delitto della consapevole consacrazione di una sola materia senza l'altra o anche l'una e l'altra fuori alla celebrazione eucaristica (cfr. Normae art. 3 §). Tale consacrazione è sempre valida, ma illecita (cfr. can. $927 \mathrm{CIC})^{28}$. Il legislatore stabilisce che tale consacrazione è assolutamente illecita anche nel caso di urgente estrema necessità, come ad esempio in pericolo di morte

25 Concilio Ecumenico Vaticano II, Costituzione dogmatica sulla Chiesa Lumen Gentium, 21 novembre 1964, AAS 57 (1965), n. 10.

26 Giovanni Paolo II, Lettera Apostolica Ordinatio Sacerdotalis sull'ordinazione sacerdotale da riservarsi soltanto agli uomini, 22 maggio 1994, AAS 86 (1994) 545-548.

27 Congregazione per la Dottrina della Fede, Normae de gravioribus delictis, art. $5,3^{\circ}$.

28 Congregazione per la Dottrina della Fede, Normae de gravioribus delictis, art. 3 §2. 
o quando non si dispone di Ostie consacrate in numero sufficiente per distribuire a tutti la Comunione, giacché in tal caso, il sacrificio è già consumato e completo ${ }^{29}$. Le Norme de gravioribus delictis prevedono sanzioni per questo delitto. Il suo autore, che può essere soltanto un sacerdote ordinato validamente, «può essere punito a secondo della gravità del crimine, non escludendo la deposizione o la dimissione dallo stato clericale» ${ }^{30}$. Egli dovrebbe essere punito sia se è ministro della consacrazione e autore del delitto, sia se consacrando le specie eucaristiche è consapevole che, dopo saranno profanate da un'altra persona.

Occorre sottolineare che anche i sacerdoti che hanno consacrato una o due materie a scopo sacrilego sono soggetti alle sanzioni penali, quand'anche sia stato loro vietato di celebrare l'Eucaristia a causa di una scomunica (cfr. can. $1331 \S 1,1^{\circ} \mathrm{CIC}$ ), interdetto (cfr. can. $1332 \mathrm{CIC}$ ) o sospensione (cfr. can. $1333 \S 1,1^{\circ} \mathrm{CIC}$ ).

\section{La sollecitazione ad turpia nella confessione}

Le Norme de gravioribus delictis tra i delicta graviora contra sancti- Canon Law tatem sacramenti Paenitentiae menzionano i delitti che prevedono la dimissione dallo stato clericale. A giudizio della CDF viene riservata «la sollecitazione al peccato contro il sesto comandamento del Decalogo nell'atto, in occasione o con il pretesto della confessione se diretta al peccato con lo stesso confessore» ${ }^{31}$.

Le altre forme di sollecitazione definite dal can. 1387 CIC non sono riservate al giudizio della $\mathrm{CDF}$, anche se prevedono la possibilità di dimissione dallo stato clericale. Il loro giudizio, con eventuale dimissio de statu clericale si svolge secondo le norme previste per il processo penale.

\section{La violazione diretta e indiretta del sigillo sacramentale}

Un altro precetto che prevede la dimissione dallo stato clericale, secondo Normae art. $4 \S 1$, n. 5 è la violazione del sigillo sacramentale. Qui si tratta di un obbligo specialissimo dei confessori, cioè presbiteri e vescovi, sia per la sua assolutezza sia per la sua massima gravità.

29 Cfr. M. Stokłosa, Ochrona Najświętszej Eucharystii, p. 219.

30 Congregazione per la Dottrina della Fede, Normae de gravioribus delictis, art. $3 \S 2$.

31 Congregazione per la Dottrina della Fede, Normae de gravioribus delictis, art. $4 \S 1,4^{\circ}$. 
Il sigillo sacramentale è «una particolarissima forma di segreto, senza dubbio la più alta e assoluta, che tocca ogni sacerdote confessore il quale non potrà mai, per nessun motivo e in alcun modo, tradire il penitente rivelando anche solo parzialmente il contenuto della confessione, così che si possa stabilire una relazione tra il peccato e chi l'ha commesso ${ }^{32}$. A causa del suo carattere inviolabile da chicchessia e irrevocabile dal depositario o da altri, tare dovere è definito col termine sigillo. Esso ha per custode Dio stesso. Si è soliti sostenere che «quanto è ascoltato nel foro di Dio, dove sempre rimane nel foro di Dio» ${ }^{33}$.

A differenza del sigillo si tratta anche del segreto sacramentale, al quale sono obbligati l'eventuale interprete e «tutti gli altri ai quali in qualunque modo sia giunta notizia dei peccati dalla confessione» (can. $983 \S 2$ CIC). Questo può avvenire per esempio «nel caso del penitente che, a motivo dell'accentuata sordità, confessi i suoi peccati con voce talmente alta, da farsi sentire a distanza dai fedeli presenti nel luogo sacro in cui è amministrato il sacramento» ${ }^{34}$.

La materia del sigillo sacramentale è trattata dal can. $983 \S 1$ CIC, dove è indicato chiaramente il soggetto tenuto al tale sigillo, che è il solo confessore. Anche se la confessione fosse invalida o per qualche ragione l'assoluzione non venisse data il sigillo deve essere mantenuto. Esso non può mai essere infranto, neanche parzialmente con i gesti, né con le parole, né con gli scritti o altri mezzi. Il segreto più assoluto deve coprire ogni peccato, mortale o veniale, occulto o pubblico, che il penitente ha confessato sia come proprio sia in collaborazione con altri, ma anche le varie espressioni del penitente nella confessione, l'eventuale negazione dell'assoluzione e il tipo di soddisfazione imposta ${ }^{35}$.

La materia appartiene al diritto divino positivo, essendo connessa chiaramente all'istituzione del sacramento (avvenuta nell'apparizione del Cristo, in Gv 20, 22-23) e in essa implicitamente contenuta ${ }^{36}$. Essa si collega anche al diritto divino naturale, che vieta sia la diffamazione sia la violazione dell'intimità che ogni persona ha il diritto di godere (cfr. can. 220 CIC).

Come è già noto, l'oggetto essenziale del sigillo sacramentale è tutto ciò che un soggetto ha dichiarato di sé nella confessione. In ambito

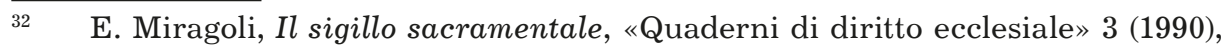
p. 411.

33 V.De Paolis, De delictis contra sanctitatem sacramenti Paenitentiae, «Periodica» 79 (1990), p. 191.

34 B. F. Pighin, Diritto penale canonico, p. 452.

35 Cfr. B. F. Pighin, Diritto sacramentale canonico, Venezia 2016, p. 290-292.

36 B. F. Pighin, Diritto penale canonico, p. 453. 
penale, secondo le norme del can. 1388 CIC, si distingue tra violazione diretta e violazione indiretta.

La violazione diretta consiste nella rivelazione di peccati dichiarati e nell'identificazione del relativo autore (il confessore deve farlo consapevolmente) $)^{37}$, ed è punita con la scomunica latae sententiae riservata alla Sede Apostolica (cfr. can. 1388 \$1 CIC). Il tribunale competente è la $\mathrm{CDF}$, come è previsto dal questo canone, «ed è ribadito sia dal m.p. SST che dalle successive Normae all'art. 5, §1, n. 5. Per effetto della riserva, occorrono venti anni perché l'azione criminale si estingua per prescrizione (cfr. can. 1362, §1, n. 1 e art $5 \S 1$, delle stesse Normae $»^{38}$. Da quel momento al confessore si applica si applica quanto è illustrato nel can. $1331 \S 1$ : in particolare, non potrà celebrare e ricevere i sacramenti. «Dovrà prima farsi togliere la scomunica, che, nel caso, è riservata alla Sede Apostolica, e pertanto da quel momento non troverà sacerdote alcuno che possa autonomamente rimettergli quella pena e dargli l'assoluzione del peccato. La scomunica pertanto potrà essere rimessa tramite la domanda che il confessore (a cui chi ha violato il sigillo si affida) inoltrerà - senza fare il nome del reo - alla Penitenzieria Apostolica» ${ }^{39}$.

La violazione indiretta ha luogo quando per leggerezza o imprudenza, senza manifestare il nome del peccatore, si crea il pericolo o si pongono le premesse cosicché altri possano sapere o sospettare qualcosa che cade sotto il sigillo sacramentale ${ }^{40}$. L'infrazione indiretta del sigillo «è sanzionata ferendae sententiae con una pena obbligatoria indeterminata $»^{41}$. Occorre, dunque «l'intervento esterno dell'autorità (il giudice o l'Ordinario), intervento che sarà provocato - bisogna supporre - dalla denuncia del penitente che si sente "tradito" o danneggiato. Siamo dunque in presenza di una pena ferendae sententiae, non determinata in maniera generale dal Codice, ma obbligatoria ${ }^{42}$.

Per quanto concerne la violazione diretta o indiretta del sigillo sacramentale, si può notare che si stratta di una profanazione di un peculiare tipo di segreto che obbliga il confessore a «non rivelare mai, per nessun motivo e senza possibilità di eccezione, il penitente $\mathrm{e}$

\footnotetext{
$37 \quad$ Cfr. M. Wronowska, Przyczyny i procedura, p. 507.

38 B. F. Pighin, Diritto penale canonico, p. 453.

$39 \quad$ E. Miragoli, Il sigillo sacramentale, p. 418.

$40 \quad$ Cfr. M. Wronowska, Przyczyny i procedura, p. 507.

$41 \quad$ B. F. Pighin, Diritto penale canonico, p. 453.

$42 \quad$ E. Miragoli, Il sigillo sacramentale, p. 418.
} 
i peccati che il penitente stesso gli abbia manifestato nel sacramento della confessione $»^{43}$.

\section{La captazione e la divulgazione delle confessioni sacramentali}

Un altro delitto contro la santità del sacramento della Penitenza consiste nella «registrazione fatta con qualunque mezzo tecnico, o nella divulgazione con i mezzi di comunicazione sociale svolta con malizia, delle cose che vengono dette dal confessore o dal penitente nella confessione sacramentale, vera o falsa. Colui che commette questo delitto, è punito secondo la gravità del crimine, non esclusa la dimissione o la deposizione» ${ }^{44}$. Questo delitto non è previsto dal Codice di Diritto Canonico dal 1983. In questa materia si applicava la dichiarazione della CDF dal 23 marzo 1973, la quale prevedeva la scomunica latae sententiae per un atto contro la santità del sacramento della penitenza compiuto attraverso la registrazione e diffusione dell'oggetto del questo sacramento ${ }^{45}$. Con la promulgazione del CIC' 83 tale dichiarazione è stata abrogata (cfr. can. $6 \$ 1,3^{\circ} \mathrm{CIC}$ ). Dopo cinque anni dalla promulgazione del codice vigente, visto il grande sviluppo dei mezzi tecnici e guardando i casi di registrazione del sacramento della Penitenza, la CDF, con decreto dal 23 settembre 1988, ha restaurato la pena del scomunica latae sententiae «per chiunque registra con qualsiasi strumento tecnico ciò che nella Confessione Sacramentale, vera o simulata, fatta da sé o da un altro, viene detto dal confessore o dal penitente, oppure lo divulga con strumenti di comunicazione sociale» ${ }^{46}$.

Autore di tali delitti può essere sia il penitente, sia il ministro sacro. Questo può succedere sia durante una confessione vera o simulata, e può anche essere fatto da una terza persona, la quale diventa autore di uno dei tre atti vietati; questo terzo può essere o laico o chierico ${ }^{47}$.

$43 \quad$ D. Cito, Delicta graviora, p. 47.

44 Congregazione per la Dottrina della Fede, Normae de gravioribus delictis, art. $4 \S 2$.

45 Cfr.M. Stokłosa,Przestępstwa przeciwko świętości sakramentupokuty w świetle kanonicznego prawa karnego, «Studia Redemptorystowskie» 7 (2009), p. 166.

46 Congregazione per la Dottrina della Fede, Declaratio de tuenda Sacramenti Paenitentiae dignitate, 23 marzo 1973, AAS 65 (1973) 678.

47 Cfr. V. De Paolis, D. Cito, Le sanzioni nella Chiesa, p. 348. 
Le Norme de gravioribus delictis stabiliscono che colui che commette tale delitto sia punito secondo la gravità del crimine, non escludendo la dimissione dallo stato clericale ${ }^{48}$.

\section{I delitti contro i costumi}

Il terzo gruppo dei crimini riservati alla $\mathrm{CDF}$ sono i delitti contro la morale. La loro fonte è il can. $1395 \$ 2$ CIC che distingue quattro tipi di reato contro il sesto comandamento del Decalogo, cioè il delitto compiuto con violenza, con minacce, pubblicamente, o con un minore al di sotto dei sedici anni sia di sesso femminile, che maschile. Considerando la natura particolarmente grave di tale crimine, il Legislatore nelle Norme de gravioribus delictis ha innalzato l'età dell'abusato da sedici a diciotto anni ${ }^{49}$.

Come vittima dell'abuso si riconosce anche una persona che abitualmente possiede un uso imperfetto della ragione. Non essendo specificata la causa di tale infermità a livello imperativo, si ritiene che essa possa dipendere sia da infermità psichica, che da infermità provocata dall'uso abituale di sostanze alcoliche o superfacenti. Inoltre, non essendo indicato un limite di età per questa ultima ipotesi, si considera rilevante anche la senilità, come nel caso della c.d. demenza senile, ovvero quando in un determinato soggetto si verifica una turbativa patologica dei normali processi intellettivi ${ }^{50}$.

Soggetto del delitto contra mores è colui che ha ricevuto almeno l'ordinazione diaconale (cfr. can. $266 \S 1$ ). Non è considerato reo chi ha perso lo stato clericale sia per sentenza giudiziaria o decreto amministrativo, sia mediante pena di dimissione irrogata legittimamente, oppure per rescritto della Sede Apostolica (cfr. can. 290). Questo crimine viene commesso indipendentemente dal comportamento della vittima. «Non è importante se un minore ha accettato questo atto, o l'ha provocato» ${ }^{51}$. Come crimine si riconosce non solo un vero e proprio rapporto sessuale tra chierico e minore del tipo di crimine di

48 Cfr. D. Cito, Nota alle nuove norme sui Delicta graviora, «Ius Ecclesiae» 22 (2010), n. 3, p. 795.

49 Cfr. Congregazione per la Dottrina della Fede, Normae de gravioribus delictis, art. $6 \S 1,1^{\circ}$.

50 C. Papale, I delitti contro la morale, [in:] AA.VV., Questioni attuali di diritto penale canonico, Studi Giuridici XCVI, Città del Vaticano 2012, p. 56.

$51 \quad$ V. De Paolis, D. Cito, Le sanzioni nella Chiesa, p. 361. 
pedofilia, ma anche l'efebofilia o l'omesessualismo ${ }^{52}$. Assume rilevanza penale anche il semplice compimento di atti impuri come ad esempio: contatti con organi genitali, toccamenti o carezze lascive, oppure di atti compiuti dal reo su se stesso, come la masturbazione o l'esibirsi nudo in presenza del minore, nonché il mostrare al minore materiale pornografico. Ciò può essere fatto mediante mezzi audiovisivi offerti da Internet (ad es. webcam) o mediante lettera o cellulare, anche come messaggio SMS o di un altro tipo ${ }^{53}$.

Lultimo crimine contro la morale è delitto in materia di reati a sfondo sessuale, ossia il così detto delitto di pedo-pornografia ${ }^{54}$. Secondo il Catechismo della Chiesa Cattolica, la pornografia consiste nel «sottrarre all'intimità dei partner gli atti sessuali, reali o simulati, per esibirli deliberatamente a terze persone. Offende la castità perché snatura l'atto coniugale, dono intimo, reciproco dei coniugi. Lede gravemente la dignità di coloro che vi si prestano, poiché l'uno diventa per l'altro oggetto di un piacere rudimentale e di un illecito guadagno. Immerge gli uni e gli altri nell'illusione di un mondo irreale; è una colpa grave. Le autorità civili devono impedire la produzione e la diffusione di materiali pornografici $»^{55}$.

Il delitto di pedo-pornografia si configura quando un chierico consegue, «con qualsiasi modalità e con qualsiasi strumento, l'acquisizione, la detenzione, o la divulgazione di immagini pornografiche aventi ad oggetto minori di anni quattordici $\aleph^{56}$. A differenza del delitto di abuso del minore, il delitto di pedo-pornografia non punisce comportamenti del reo positivamente intesi al coinvolgimento di un minore in attività di meretricio e pornografia, ma è esclusivamente finalizzato alla punizione del consumatore di quest'ultima ${ }^{57}$.

Il trattamento sanzionatorio è il medesimo per entrambe le fattispecie criminose, poiché l'acquisizione, la detenzione e la divulgazione di materiale pedopornografico, oltre a delineare la condotta gravemente

52 Cfr. E. Parolari, Aspetti psicologici dei delitti canonici, [in:] AA.VV., Questioni attuali di diritto penale canonico, Studi Giuridici XCVI, Città del Vaticano 2012, p. 69.

53 Cfr. C. Papale, I delitti contro la morale, p. 57.

Cfr. Congregazione per la Dottrina della Fede, Normae de gravioribus delictis, art. $6 \S 1$.

55 Catechismo della Chiesa Cattolica, Città del Vaticano 1992, n. 2345.

56 Congregazione per la Dottrina della Fede, Normae de gravioribus delictis, art. $6 \S 1,1^{\circ}$.

57 Cfr. E. Parolari, Aspetti psicologici dei delitti canonici, p. 67. 
riprovevole del reo, ha gravi conseguenze a livello sociale ${ }^{58}$. Tali comportamenti criminosi tendono, infatti, a incrementare la distribuzione e il commercio via Internet delle immagini di minori, consolidando una comunità di consumatori perversi ${ }^{59}$.

Per maggior precisione si può notare che «il delitto di pedo-pornografia richiede la presenza di un dolo specifico in capo al reo, affinché si configuri tale fattispecie penale, infatti, occorre che il chierico abbia agito a scopo libidine ${ }^{60}$.

La prescrizione per l'esercizio dell'azione penale in caso di tali delitti è diversa. «Per l'abuso di minore è di vent'anni che decorrono a partire dal compimento del diciottesimo anno di età della vittima» ${ }^{61}$. Per quanto riguarda, invece, il delitto di pedo-pornografia, «il termine è il medesimo, ma decorre dal giorno in qui è stato commesso il delitto, poiché non è possibile risalire a tutte le relative date di nascita dei minori ritratti nelle immagini ${ }^{62}$.

Per quanto riguarda gli aspetti procedurali, qualora rilevino i delitti di cui sopra, la competenza in via preliminare spetta ai Vescovi diocesani o ai Superiori Maggiori. Se l'accusa del crimine appare verosimile, l'Ordinario del luogo o personalmente, o mediante il suo delegato procede con un'indagine previa secondo quanto disposto dal can. 1717 CIC. Terminata quest'ultima, se l'accusa appare fondata, il caso deve essere sottoposto all'esame della CDF, che ha competenza esclusiva in materia ${ }^{63}$.

«Le sanzioni canoniche applicate nei confronti di un chierico colpevole di abuso di minore, persona che abitualmente possiede un uso imperfetto della ragione, o di delitto di pedo-pornografia, sono misure restrittive che aboliscono l'esercizio del suo ministero pubblico in modo completo, eliminando ogni tipo di contatto con minori» ${ }^{64}$. Tali

$58 \quad$ Cfr. M. Stokłosa, Utrata stanu duchownego, p. 143.

$59 \quad$ Cfr. C. Papale, I delitti contro la morale, p. 57.

${ }_{60}$ Congregazione per la Dottrina della Fede, Normae de gravioribus delictis, art. $6 \S 1,2^{\circ}$.

${ }_{61}$ Congregazione per la Dottrina della Fede, Normae de gravioribus delictis, art. $6 \S 1,1^{\circ}$.

62 C. Papale, I delitti contro la morale, p. 65.

${ }_{63}$ Cfr. Ch. J. Scicluna, Delicta graviora. Ius processuale, [in:] AA.VV., Questioni attuali di diritto penale canonico, Studi Giuridici XCVI, Città del Vaticano 2012, p. 79-94.

64 A. D'Auria, La scelta della procedura per l'irrogazione delle pene, [in:] AA.VV., Questioni attuali di diritto penale canonico, Studi Giuridici XCVI, Città del Vaticano 2012, p. 113-133. 
misure possono essere accompagnate da un precetto penale o da pene canoniche (cfr. can. $1395 \S 2$ CIC), fra cui la più grave è la dimissione dallo stato clericale ${ }^{65}$.

\section{PRZYCZYNY USUNIĘCIA ZE STANU DUCHOWNEGO WEDEUG KOMPETENCJI KONGREGACJI NAUKI WIARY}

Artykuł porusza zagadnienie przeniesienia duchownych do stanu świeckiego według norm De Gravioribus Delictis. Zostały w nim omówione poszczególne przestępstwa, których popełnienie przewiduje utratę stanu duchownego, a których osąd zarezerwowany jest wyłącznie Kongregacji Nauki Wiary. W pierwszym paragrafie zostały opisane przestępstwa przeciwko wierze i jedności Kościoła, takie jak apostazja, herezja i schizma. Paragraf drugi porusza zagadnienie uzurpacji urzędów kościelnych i nadużyć w wykonywaniu władzy święceń. Mówi się tutaj o przestępstwach popełnionych na polu sakramentów Kościoła. Ostatni, trzeci paragraf porusza problem przestępstw dotyczących obyczajów, które również przewidują karę przeniesienia duchownych do stanu świeckiego.

Słowa klucze: utrata stanu duchownego, przestępstwo ciężkie, profanacja, solicytacja, nadużycie władzy święceń.

\section{Bibliografia:}

1. Catechismo della Chiesa Cattolica, Città del Vaticano 1992.

2. Chiappetta L., Il Codice di Diritto Canonico. Commento giuridico-pastorale, vol. I-III, Bologna 2011.

3. Cito D., Delicta graviora contro la fede e sacramenti, [in:] AA.VV., Questioni attuali di diritto penale canonico, Studi Giuridici XCVI, Città del Vaticano 2012.

4. Cito D., Nota alle nuove norme sui Delicta graviora, «Ius Ecclesiae» 22 (2010), n. 3.

5. Concilio Ecumenico Vaticano II, Costituzione dogmatica sulla Chiesa Lumen Gentium, 21 novembre 1964, AAS 57 (1965).

6. Congregazione dei Vescovi, Dichiarazione della scomunica di mons. M. Lefebvre, EV 11 (1988-1989), 692-694.

7. Congregazione per la Dottrina della Fede, Declaratio de associationibus massonicis, 26 novembre 1983, AAS 76 (1984).

8. Congregazione per la Dottrina della Fede, Declaratio de tuenda Sacramenti Paenitentiae dignitate, 23 marzo 1973, AAS 65 (1973).

65 Cfr. Congregazione per la Dottrina della Fede, Normae de gravioribus delictis, art. $6 \S 2$. 
9. Congregazione per la Dottrina della Fede, Normae de gravioribus delictis Congregationi pro Doctrina Fidei reservatis seu Normae de delictis contra fidem necnon de gravioribus delictis, 21 maggio 2010, AAS 102 (2010).

10. De Paolis V., Cito D., Le sanzioni nella Chiesa. Commento al Codice di Diritto Canonico. Libro VI, Città del Vaticano 2000.

11. De Paolis V., De delictis contra sanctitatem sacramenti Paenitentiae, «Periodica» 79 (1990).

12. Dezzuto C., Delicta reservata contro la fede e contro $i$ sacramenti, [in:] AA.VV., I delitti riservati alla Congregazione per la Dottrina della Fede, Quaderni di Ius Missionale, Città del Vaticano 2014.

13. D'Auria A., La scelta della procedura per l'irrogazione delle pene, [in:] AA.VV., Questioni attuali di diritto penale canonico, Studi Giuridici XCVI, Città del Vaticano 2012.

14. Ferrante M., Il delitto del apostasia alla luce del Motu Proprio "Omnium in mentem", [in:] AA.VV., Questioni attuali di diritto penale canonico, Studi Giuridici XCVI, Città del Vaticano 2012.

15. Giovanni Paolo II, Costituzione Apostolica Pastor Bonus, 28 giugno 1988, AAS 80 (1988).

16. Giovanni Paolo II, Lettera Apostolica Ordinatio Sacerdotalis sull'ordinazione sacerdotale da riservarsi soltanto agli uomini, 22 maggio 1994, AAS 86 (1994).

17. Marzoa A., Commento al c. 1364, [in:] A. Marzoa, J. Miras, R. Rodríguez-Ocaña (a cura di), Comentario exegético al Código de Derecho Canónico, vol. IV/1, Pamplona 2002.

18. Miragoli E., Il sigillo sacramentale, «Quaderni di diritto ecclesiale» 3 (1990).

19. Papale C., I delitti contro la morale, [in:] AA.VV., Questioni attuali di diritto penale canonico, Studi Giuridici XCVI, Città del Vaticano 2012.

20. Parolari E., Aspetti psicologici dei delitti canonici, [in:] AA.VV., Questioni attuali di diritto penale canonico, Studi Giuridici XCVI, Città del Vaticano 2012.

21. Pawluk T., Prawo kanoniczne wedtug Kodeksu Jana Pawła II, vol. IV, Olsztyn 1990.

22. Pighin B. F., Diritto penale canonico, Venezia 2014.

23. Pighin B. F., Diritto sacramentale canonico, Venezia 2016.

24. Scicluna Ch. J., Delicta graviora. Ius processuale, [in:] AA.VV., Questioni attuali di diritto penale canonico, Studi Giuridici XCVI, Città del Vaticano 2012.

25. Stokłosa M., Ochrona Najświętszej Eucharystii w świetle motu proprio Jana Pawta II Sacramentorum santitatis tutela, «Studia Redemptorystowskie» 8 (2010).

26. Stokłosa M., Przestępstwa przeciwko świętości sakramentu pokuty w świetle kanonicznego prawa karnego, «Studia Redemptorystowskie» 7 (2009).

27. Syryjczyk J., Apostazja od wiary w świetle przepisów kanonicznego prawa karnego. Studium prawno-historyczne, Warszawa 1984.

28. Syryjczyk J., Kanoniczne prawo karne. Część szczegółowa, Warszawa 2003.

29. Syryjczyk J., Profanacja Eucharystii wedtug ustawodastwa kanonicznego i polskiego prawa karnego, «Prawo Kanoniczne» 29 (1986), n. 3-4.

30. Wronowska M., Przyczyny i procedura wydalenia ze stanu duchownego, «Studia Ełckie» 16 (2014), n. 4. 